\title{
Medical and Surgical Management of a Descending Aorta Penetrating Atherosclerotic Ulcer and Associated Ascending Intramural Hematoma
}

\author{
Matthew C. Henn, MD ${ }^{1}$, Christopher P. Lawrance, $\mathrm{MD}^{1}$, Alan C. Braverman, MD², \\ Luis Sanchez, $\mathrm{MD}^{3}$, Jennifer S. Lawton, $\mathrm{MD}^{1 *}$ \\ ${ }^{1}$ Division of Cardiothoracic Surgery, Department of Surgery, Washington University School of Medicine, Barnes-Jewish Hospital, \\ St. Louis, Missouri; ${ }^{2}$ Division of Cardiology, Department of Medicine, Washington University School of Medicine, Barnes-Jewish \\ Hospital, St. Louis, Missouri; and ${ }^{3}$ Division of Vascular Surgery, Department of Surgery, Washington University School of Medicine, \\ Barnes-Jewish Hospital, St. Louis, Missouri
}

\begin{abstract}
A 69-year-old man presented with chest pain and a computed tomography scan demonstrated an acute penetrating atherosclerotic ulcer (PAU) of the proximal descending aorta with an associated intramural hematoma (IMH) extending retrograde to the aortic root and distally to the renal arteries. He successfully underwent endovascular repair of the PAU and medical management of the associated ascending intramural hematoma with complete resolution at 6 months.

Copyright @ 2014 Science International Corp.
\end{abstract}

\section{Key Words}

Penetrating aortic ulcer - Ascending intramural hematoma - TEVAR

\section{Introduction}

Penetrating aortic ulcers (PAUs) are well defined entities that are characterized by a disruption of the internal elastic lamina of the aorta that penetrates through the intima into the media. A hallmark of a PAU is the absence of an intimal flap (as is routinely seen with a dissection) and it is often associated with an intramural hematoma (IMH). Ulcerations are typically localized in areas with extensive amounts of atherosclerosis and calcification. PAUs can penetrate through the media and into the adventitia, resulting in pseudoaneurysm formation or free-wall rupture [1].

Management of PAUs and associated IMHs classically depends on the location of the pathology, but whether medical management or surgical intervention is preferred remains controversial. Generally, PAUs of the ascending aorta with associated IMH are considered surgical emergencies because of their malignant nature and risk of rupture and tamponade [2]. Acute IMH of the ascending aorta is usually treated with urgent cardiac surgery [3]. However, management of PAUs in the descending thoracic aorta with associated IMH of the ascending aorta remains unclear [4]. We present a case report of successful management of a descending thoracic PAU and ascending aortic IMH with endovascular repair of the PAU and medical management coupled with serial surveillance of the ascending aortic IMH.

\section{Case Description}

A 69-year-old Caucasian gentleman with a past medical history of uncontrolled hypertension, mild chronic kidney disease, paroxysmal atrial fibrillation,

\footnotetext{
*Corresponding author:

Jennifer Lawton, MD

Division of Cardiothoracic Surgery

Department of Surgery

Washington University School of Medicine

660 S. Euclid Avenue, Campus Box 8234

St. Louis, MO 63110

Tel: +1 314362 2821, Fax: +1 314747 4216, E-Mail: lawtonj@wustl.edu
} 


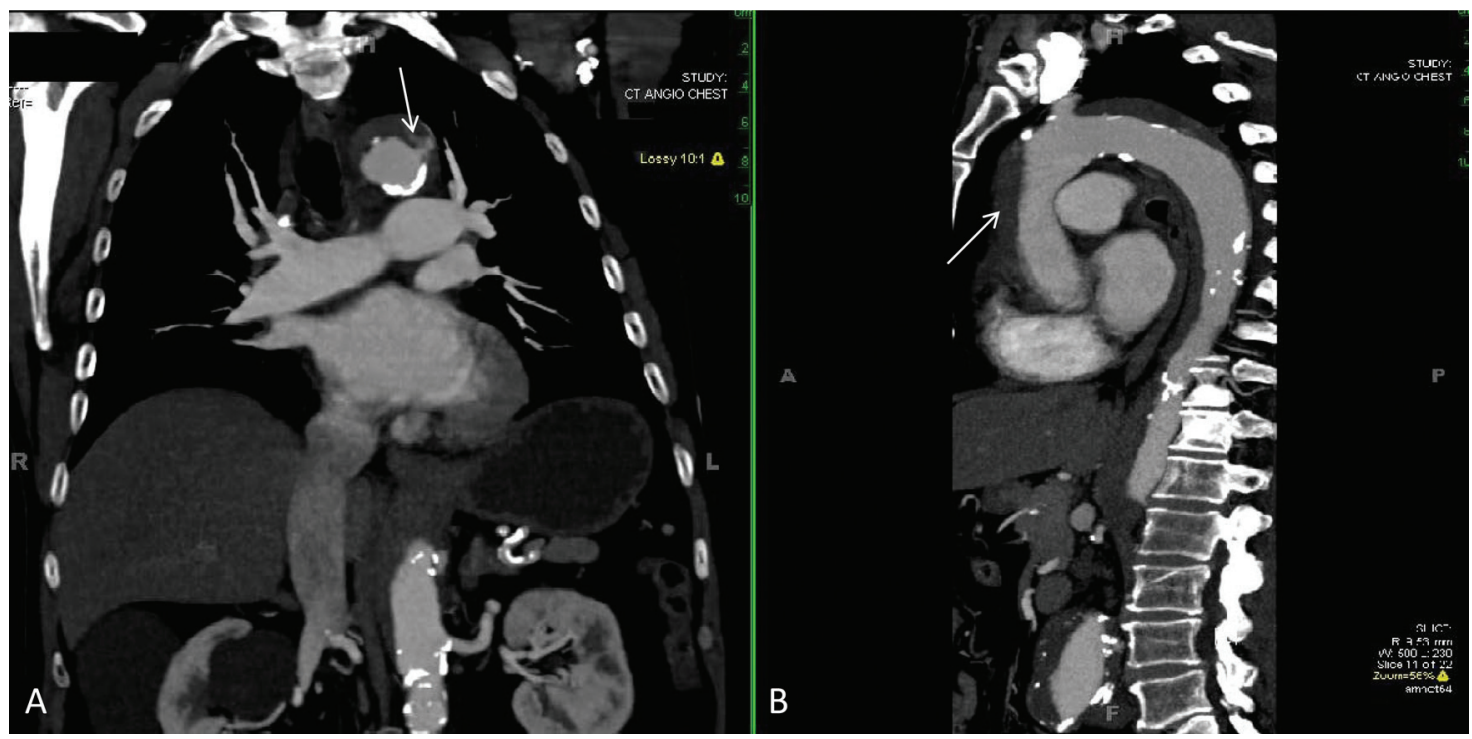

Figure 1. Computed tomographic angiogram (CTA) of the chest, abdomen, and pelvis at the time of presentation. (A) Coronal section demonstrating penetrating atherosclerotic ulcer with active extravasation of contrast into the wall of the aorta. (B) Sagittal section demonstrating intramural hematoma of the aorta extending from the aortic valve to the celiac branches.

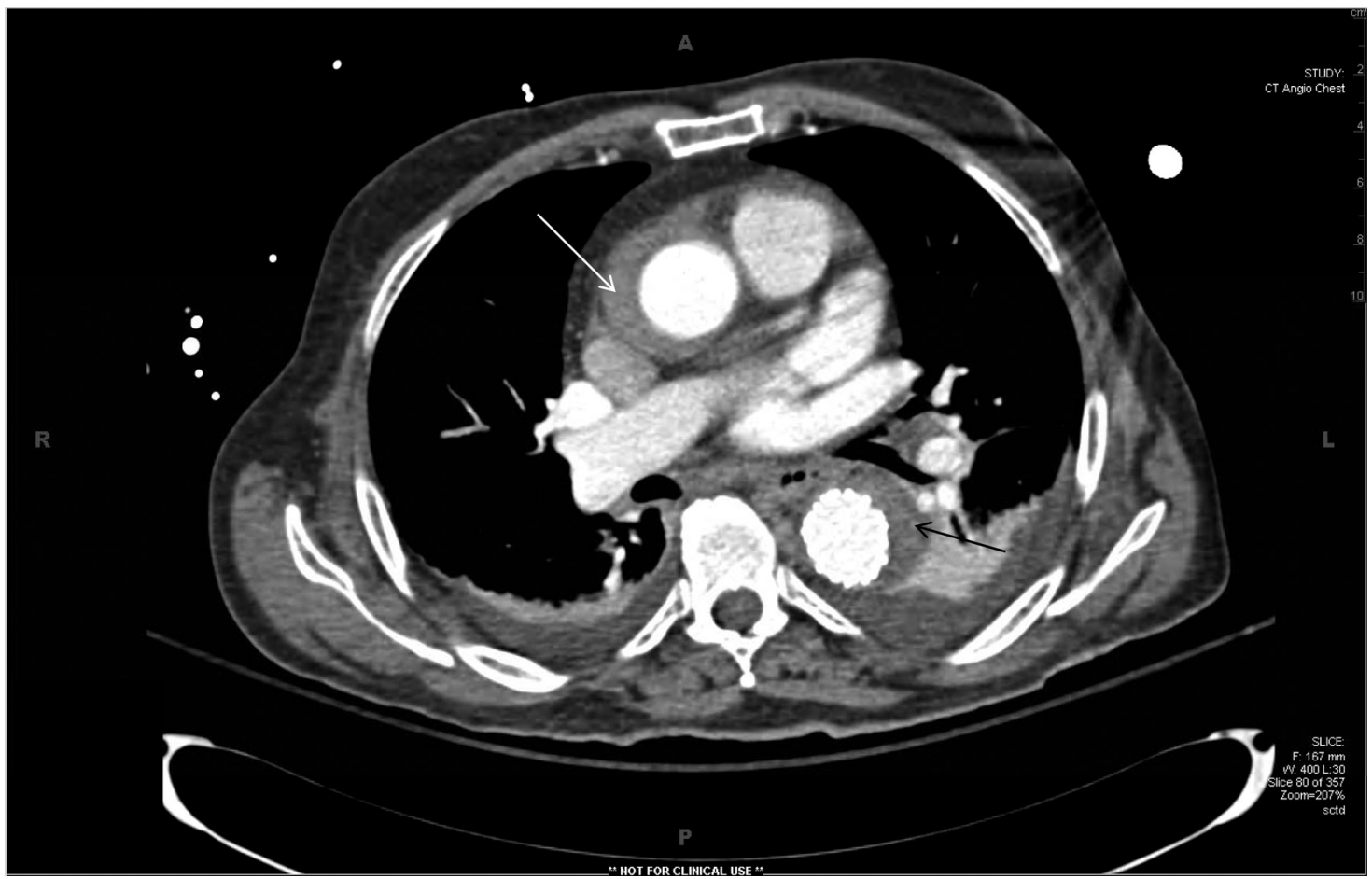

Figure 2. Computed tomographic angiogram (CTA) of the chest on postoperative day 2 demonstrating stable intramural hematoma (white arrow) and endoleak (black arrow).

peripheral vascular disease, and known $5.7 \mathrm{~cm}$ abdominal aortic aneurysm presented to the emergency room with 24 hours of sudden-onset chest and epigastric pain and one episode of hematemesis. A computed tomography angiogram (CTA) of the chest, abdomen, and pelvis demonstrated a penetrating aortic ulcer $3-4 \mathrm{~cm}$ distal to the origin of the left subclavian artery with active extravasation of contrast into the media, with associated large intramural hematoma that extended retro- 


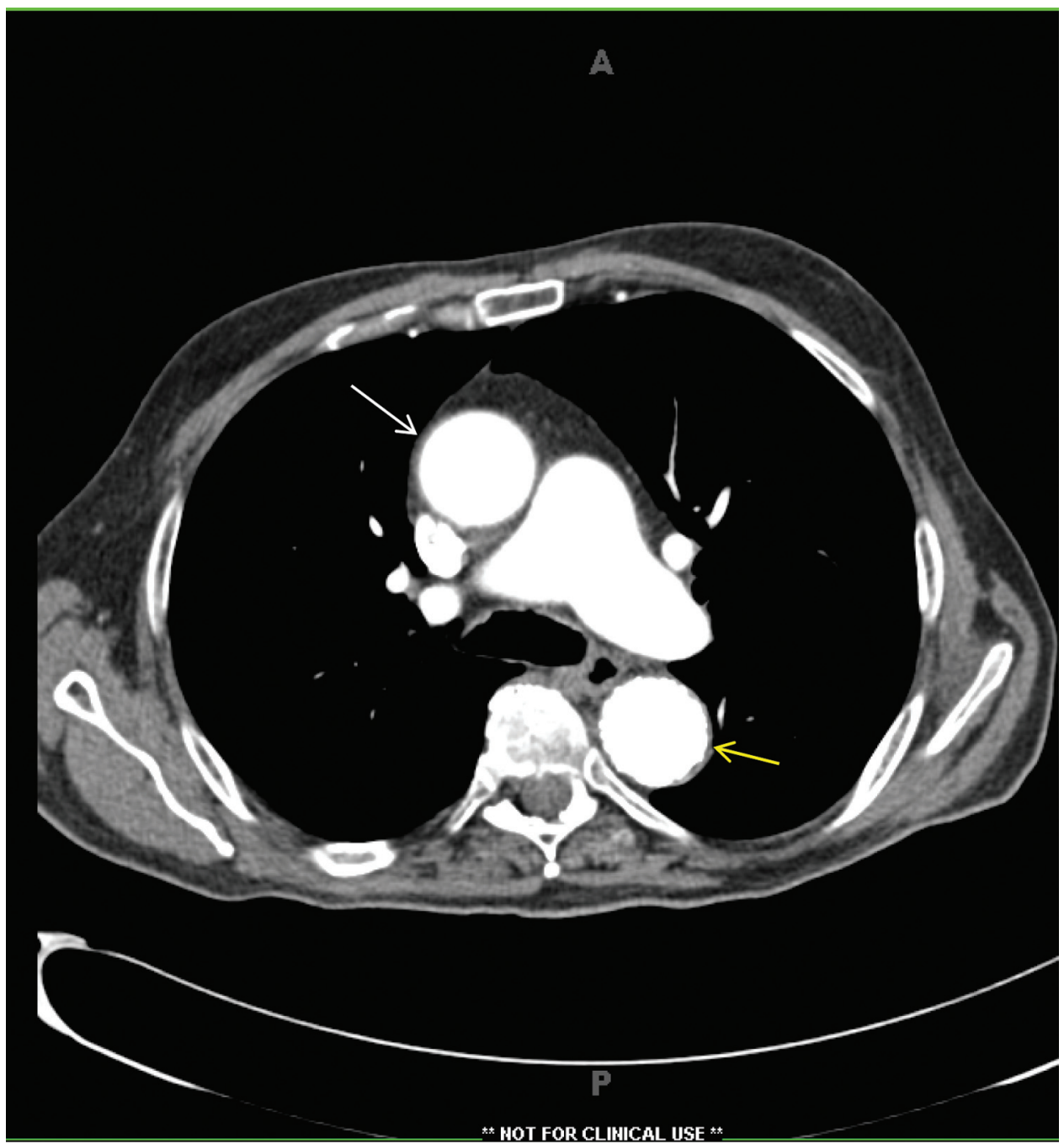

Figure 3. Seven month follow-up computed tomographic angiogram (CTA) of the chest demonstrating complete resolution of intramural hematoma (white arrow) and resolved endoleak (yellow arrow).

grade to the aortic root and distally to the origins of the renal arteries (Fig. 1).

The patient was taken to the operating room and underwent thoracic endovascular aortic repair (TEVAR) via bilateral femoral arterial approach. A 37 $\mathrm{mm} \times 15 \mathrm{~cm}$ TAG endovascular graft (Gore, Flagstaff, AZ, USA) was placed that partially occluded the left subclavian artery and extended distally over the penetrating aortic ulcer. Postplacement angiogram demonstrated that all other branches of the aorta were widely patent and the ulcer was completely excluded. Postoperatively, the patient was monitored in the intensive care unit with aggressive blood pressure control.
On postoperative day 2 , the patient underwent repeat $\mathrm{CT}$ scan of his chest, abdomen, and pelvis that demonstrated persistent intramural contrast seen adjacent to the penetrating ulcer, which extended distally within the distal descending thoracic aorta on the more delayed images signifying a type III endoleak. The intramural hematoma persisted from the aortic root to the celiac axis and was stable in size (Fig. 2). At this point, it was decided to continue to observe the patient clinically and forgo operative repair of his ascending aortic intramural hematoma.

The rest of the patient's postoperative course was uneventful, and he underwent repeat CTA of the 
chest, abdomen, and pelvis 2 weeks after surgery. This demonstrated complete resolution of the endoleak. The intramural hematoma involving the ascending and descending thoracic aorta had decreased in attenuation. The patient was then discharged home and underwent follow up CTA at 1 month, which demonstrated a reduction in thickness of the ascending $\mathrm{IMH}$. Follow-up CTA at 7 months demonstrated complete resolution of the intramural hematoma (Fig. 3).

\section{Discussion}

With the widespread use of TEVAR, symptomatic, high-risk PAUs of the descending aorta are generally repaired via an endovascular approach due to the localized nature of the disease [5]. However, PAUs with associated IMHs present challenges for the TEVAR approach including friable, unhealthy landing zones for the endograft, which may compromise the long-term results [5]. For this reason, some argue that waiting for $\mathrm{IMH}$ resolution in stable patients provides better longterm success rates [5]. To date, there has been no randomized comparison in the literature to support either approach.

While emergency open surgery is generally indicated for appropriate candidates in ascending IMHs [3], there is experience from several Asian studies with relatively low early mortality rates with initial medical therapy for certain patients [5-7]. Interestingly, one study reported freedom from IMH-related events including dissection, tamponade, and rupture as $65 \%$ at 5 years with a 5 year survival rate of $80 \%$ to $85 \%$, which was comparable to that of descending IMHs [6]. However, among Western studies of acute ascending $\mathrm{IMH}$, morbidity and mortality remain high with initial medical therapy, including development of cardiac tamponade and progression to classic aortic dissection [3]. Thus, surgical management of acute ascend- ing IMH is generally recommended in North American and European centers. Descending IMHs are generally treated medically unless complications occur, with complete resorption of the IMH reported in up to $50 \%$ of cases [3].

In our case, by sealing the acutely leaking penetrating aortic ulcer and preventing further extravasation of blood into the aortic wall, we theorized that the resultant ascending IMH may stabilize and eventually resolve.

\section{Conclusion}

Here we present a case with successful management of a PAU of the descending aorta with associated ascending IMH utilizing a hybrid approach. With operative repair of the PAU via an endovascular approach and medical management of the associated ascending $\mathrm{IMH}$, the patient had successful resolution of the IMH and adequate long-term positioning of his endovascular graft. While management of these complicated problems remains controversial, this case provides evidence that a multidisciplinary team should determine a treatment plan that is individualized to each patient in order to obtain a successful outcome.

\section{Acknowledgments}

Support was provided by NIH T32 HL007776 and NIH RO1 HL098182-01A1.

\section{Conflict of Interest}

The authors have no conflict of interest relevant to this publication.

\section{Comment on this Article or Ask a Question}

\section{References}

1. Cooke JP, Kazmier FJ, Orszulak TA. The penetrating aortic ulcer: pathologic manifestations, diagnosis, and management. Mayo Clin Proc. 1988;63: 718-725. 10.1016/S0025-6196(12)65534-7

2. Coady MA, Rizzo JA, Hammond GL, Pierce JG, Kopf GS, Elefteriades JA. Penetrating ulcer of the thoracic aorta: what is it? How do we recognize it? How do we manage it? J Vasc Surg. 1998;27: 1006-1015; discussion 1015-1006. 10.1016/S07415214(98)70003-5
3. Harris KM, Braverman AC, Eagle KA, Woznicki EM, Pyeritz RE, Myrmel T, et al. Acute aortic intramural hematoma: an analysis from the International Registry of Acute Aortic Dissection. Circulation. 2012;126: S91-96. 10.1161/CIRCULATIONAHA.111. 084541

4. Watanabe S, Hanyu M, Arai Y, Nagasawa A. Initial medical treatment for acute type a intramural hematoma and aortic dissection.
Ann Thorac Surg. 2013;96:2142-2146. 10.1016/ j.athoracsur.2013.06.060

5. Patel HJ, Williams DM, Upchurch GR Jr, Dasika $\mathrm{NL}$, Deeb GM. The challenge of associated intramural hematoma with endovascular repair for penetrating ulcers of the descending thoracic aorta. J Vasc Surg. 2010;51:829-835. 10.1016/j.jvs.2009.11.050

6. Moizumi Y, Komatsu T, Motoyoshi N, Tabayashi K. Clinical features and long-term outcome of type $A$ 
and type B intramural hematoma of the aorta. J Thorac Cardiovasc Surg. 2004;127:421-427. 10. 1016/j.jtcvs.2003.09.016

7. Song JM, Kim HS, Song JK, Kang DH, Hong MK, Kim JJ, et al. Usefulness of the initial noninvasive imaging study to predict the adverse outcomes in the medical treatment of acute type a aortic intramural hematoma. Circulation.2003;108Suppl1:||324-II328.10.1161/ 01.cir.0000087651.30078.38
Cite this article as: Henn MC, Lawrance CP, Braverman AC, Sanchez L, Lawton JS.Medical and Surgical Management of a Descending Aorta Penetrating Atherosclerotic Ulcer and Associated Ascending Intramural Hematoma. Aorta 2014;2(2):77-81. DOI: http://dx.doi.org/10.12945/ j.aorta.2014.13-060 